\title{
Sraffa and Goodwin: \\ A Unifying Framework for Standards of Value in the Income Distribution Problem
}

\author{
Albert E. Steenge \\ Received November 30, 1994; revised version received April 18, 1995
}

In this paper we show that direct links exist between Sraffian and Goodwinian methodologies in income distribution theory. In fact, Sraffa's Standard commodity approach and Goodwin's transformation of axes are shown to be different manifestations of the same structural approach. Both are brought under a unified framework in terms of transformations involving a transformation matrix of rank one. To obtain transparency Sraffa is shown to retain as much interdependency as possible while Goodwin sacrifies all interdependent structure. It appears that a central role is played by models related to closed models of input-output type.

\section{Introduction}

Both Sraffa and Goodwin have written extensively about issues in income distribution theory. Sraffa, to simplify the analysis, proposed a framework involving a new type of value standard, the Standard commodity. In the subsequent literature Sraffa's approach was shown to be easily modelled in terms of the right-hand Perron-Frobenius eigenvector of his system's input coefficients matrix (definitions will follow below). 'Goodwin, later on, discussed the same problem, but in terms of an approach involving all eigenvectors of the input coefficients matrix. Connections between the two approaches have - as far as we know never been explored. Yet, as we shall try to show, intimate connections between them exist. At the same time we shall see that direct links exist with results in distribution theory involving the left-hand Perron-

1 Although Sraffa did not employ the tools of modern mathematical analysis, he was in contact with leading mathematicians such as A.S. Besicovitch, F. Ramsey, and A. Watson. 
Frobenius eigenvector of the input coeffcients matrix. Below we shall, as a common denominator, focus on the role of eigenvectors serving as value standards. As we shall see, to introduce these vectors, it is usefu? first to go back to the traditional closed static Leontief input-output model. Only hereafter we shall encounter the open models. This may look surprising because Sraffa and Goodwin have presented their ideas for open static input-output models.

Below we shall start with Sraffa and concentrate on the familiar linear income distribution discussion. After having introduced eigenvectors, our strategy will be to express the role of the Sraffian Standard commodity in terms of a simple transformation matrix. Next, we shall turn to Goodwin's insights regarding a change of coordinate axes and show that his transformations are identical to ours, mutatis mutandis.

\section{The Sraffian Model}

Central in our discussion is the Sraffian price model ${ }^{2}$

$$
p=(1+r) p A+w^{*} l \text {. }
$$

Here $p$ stands for the vector of prices, $A$ for the matrix of input coefficients, $l$ for the vector of direct labor coefficients, $r$ for the uniform rate of profits, and $w^{*}$ for the wage rate measured as a share of the national income, so $0 \leq w^{*} \leq 1$. Our real output model is

$$
x=A x+f .
$$

with $x$ total output and $f$ final demand. Following Sraffa, we shall concentrate on the situation where the relation between $r$ and $w^{*}$ becomes linear

$$
r=\left(1-w^{*}\right) R .
$$

Here $R$, the maximal rate of profits, is determined by $\mu_{1}$, the PerronFrobenius eigenvalue of $A$, via

$$
R=\frac{1-\mu_{1}}{\mu_{1}} .
$$

Throughout (observed) input-output coefficients matrices are assumed to be indecomposable, primitive, and of full rank. ${ }^{3}$

2 Note that in a number of texts, the model is presented in a transformed form in which the price vector appears as a column vector. 
Standardization is required by now. The Standard system is a real output system where the final demand vector is proportional to the vector of aggregate intermediate inputs, i.e., $f$ is proportional to $A x$ in (2). This implies that both $f$ and $x$ have the proportions of the righthand Perron-Frobenius eigenvector of matrix $A$. Denoting vectors with this property by an asterisk, we have

$$
x^{*}=A x^{*}+f^{*} .
$$

where $f^{*}$ is known as the Standard commodity. We now put the value of the Standard commodity at unity, so $p f^{*}=1$. Similarly, the size of the total labor force, $l x$ (or $l x^{*}$ in the case of the Standard system), will be put at unity. Vectors $A x^{*}$ and $f^{*}$ having the proportions of the right-hand Frobenius eigenvector of $A$, the traditional proof of (3) is as follows (see, e.g., Pasinetti, 1977, p. 115). Starting from

$$
p f^{*}=p(I-A) x^{*}=1
$$

and

$$
l x^{*}=1,
$$

post-multiplication of (1) by $x^{*}$ gives

$$
p x^{*}=p A x^{*}+r p A x^{*}+w^{*} l x^{*} .
$$

So

$$
\begin{aligned}
r p A x^{*} & =p x^{*}-p A x^{*}-w^{*} J x^{*} \\
& =p(I-A) x^{*}-w^{*} \\
& =1-w^{*} .
\end{aligned}
$$

But

$$
\begin{aligned}
R p A x^{*} & =p x^{*}-p A x^{*} \\
& =p(J-A) x^{*} \\
& =1 .
\end{aligned}
$$

So

$$
\left(1-w^{*}\right)=\frac{r}{R}\left(R p A x^{*}\right),
$$

3 We should remark that Sraffa's works in fact are of a much broader nature than can be discussed here, involving joint production effects, fixed capital, and nonconstant returns as well. For the moment, however, we are concerned with single product models involving only circulating capital. Later work will have to extend our framework in terms of joint production and other variants. For issues involved in such extensions, see Steenge (1990b) or Konijn and Steenge (1995). 
or

$$
\left(1-w^{*}\right) R=r .
$$

Although the above derivations are correct, they only provide limited insight in the issues at hand. For example, no special attention is paid to implications for the price proportions over the entire range of the distributive variables $r$ and $w^{*}$. Also the relation to other work in this area is not easily established which invokes the danger of Sraffa's insight remaining rather isolated from other work in this area. To provide further insight, below we shall start with discussing a closed model. (Regarding this choice, see also the remark on Quandt, 1961, in Sect. 8.)

\section{Commodity Bundles as Standards of Value in Closed Models}

A standard of value is a special commodity the amount of which "embodied in" or "imputed to" each good or service in the system we are studying determines the system's prices or price proportions. A large literature is available on the role of standards of value (usually labor or capital) in static open Leontief models. Innovations have been proposed in several directions, e.g., in terms of models incorporating output coefficients matrices of the von Neumann type (Abraham-Frois and Berrebi, 1979). Francis Seton's (1985) combination of "valuation by cost" and "valuation by use" aspects resulted in a new weighing of the contributions of factors in terms of his "eigenprices." Standards for closed models, however, have received hardly any attention thus far. This is a pity as closed models appear to play a key role in understanding the role of other constructs such as eigenvectors (Steenge 1986, 1990a). Therefore, let us start with the closed Leontief model. Following Pasinetti (1977) or Abraham-Frois and Berrebi (1979) we thus have

$$
z=M z
$$

for the real part, and

$$
p=p M
$$

for the price side, where $z$ and $p$ stand for the equilibrium output and price vectors, and $M$ for an $n \times n$ input coefficients matrix with PerronFrobenius eigenvalue equal to unity. ${ }^{4}$ We immediately note that $z$ and $p$ are, respectively, right- and left-hand Perron-Frobenius eigenvectors of $M$.

4 We again use the symbol $p$ for the vector of prices here, since no confusion will arise. 
Clearly, the elements of matrix $M$ stand for the direct or "first order" inputs necessary for unit production of each commodity. However, we also have an interpretation for the dual systems $z=M^{2} z$ and $p=p M^{2}$; matrix $M^{2}$ gives the "second order" inputs, i.e., the inputs necessary for the production of the inputs of the first round. Continuing, we also have an interpretation for the dual systems $z=M^{3} z$ and $p=p M^{3}$, et cetera. Taking limits we obtain the systems

$$
z=M^{\infty} z
$$

and

$$
p=p M^{\infty},
$$

where the elements of the rank one matrix $M^{\infty}$ stand for the "infinite order" or "all-in" input coefficients for unit production of each commodity. As $M$ is indecomposable and primitive, we have $M^{\infty}>0.5$ All columns of $M^{\infty}$ being proportional to $z$, we see that inputs in (8) are given in terms of a well-determined scalar multiple of the output vector $z$. Likewise, from (9) we see that commodity prices are given in terms of the price of the same well-determined scalar multiple of $z$. Because all prices are expressed in terms of scalar multiples of the same commodity bundle $z$, this bundle, in this sense, strajghtforwardly can be interpreted as a system standard of value. That is, we can view each price as being expressed in terms of (the price of) a commodity bundle having the proportions of the right-hand Perron-Frobenius eigenvector of $M$.

We may obtain the same connection via another approach, i.e., via spectral decomposition properties. ${ }^{6}$ Vectors $z$ and $p$ being, respectively, right- and left-hand Perron-Frobenius eigenvectors of $M$, we straightforwardly have

5 From Markov theory we have that matrix $M^{\infty}$ can be written as the standardized outer product of (scalar multiples of) $z$ and $p$.

6 In spectral decomposition form, a matrix $A$ is written as the sum $A=$ $\mu_{1} A_{1}+\mu_{2} A_{2}+\ldots+\mu_{n} A_{n}$, where the $\mu_{i}$ are the eigenvalues of $A$ and the constituent matrices $A_{i}=v_{i} w_{i}$ the outer products of the bi-orthonormalized right-hand $\left(v_{i}\right)$ and left-hand $\left(u_{i}\right)$ eigenvectors of $A$. Properties of the $A_{i}$ include $\sum_{i=1}^{n} A_{i}=l, A_{i}^{2}=A_{i}$, and $A_{i} A_{j}=0$ for $i \neq j$. For simplicity, we assume that all eigenvalues are simple. If this is not the case, more advanced techniques must be used. This does not affect the main argument, however. If $A$ is an indecomposable input-output matrix (with Frobenius eigenvalue $\mu_{1}$ ), its left- and right-hand eigenvectors are strictly positive. Denoting them, after standardization, by $p^{*}$ and $f^{*}$, we then have $A_{1}=\left[f^{*} p^{*}\right]$, with $p^{*} f^{*}=1$. 


$$
z=M_{1} z
$$

and

$$
p=p M_{1}
$$

with $M_{1}>0$ the standardized outer product of both eigenvectors (i.e., the constituent matrix associated with the Perron-Frobenius eigenvalue of $M$ ). Clearly, $M_{1}=M^{\infty}$, so also the system given by Eqs. (10) and (11) can be interpreted as representing a closed input-output system written in terms of a Perron-Frobenius eigenvector serving as a standard of value. Note that from our original system (6)-(7) we can obtain (8)-(9) or (10)-(11) by pre- or postmultiplying with $M_{1}$; for example, from (7) we have $p M_{1}=p M M_{1}$. But $p M_{1}=p$, so (11) results straightforwardly. In this price system, therefore, simple postmultiplication results in the required expression. We may observe that similar relations exist for vectors having the proportions of the remaining eigenvectors of $M$.

So, a right-hand Perron-Frobenius eigenvector can be straightforwardly introduced as a standard of value for a closed input-output model. But how about open models? Sraffa (as shown later) and Goodwin explicitly introduce a specific concept (eigenvectors) in open Leontief-type models to obtain linear income relations. Before turning to that problem, we shall first consider a well-known special case of an open model, i.e., the case where the direct labor input coefficients vector $l$ in (1) is proportional to the left-hand Frobenius eigenvalue of $A$.

\section{Commodity Bundles as Standards of Value in a Special Case}

Let us assume for the moment that the vector of labor input coefficients $l$ in (1) has the proportions of the left-hand Frobenius eigenvector of $A$. In that case also the price vector $p$ is an eigenvector of $A$. In this particular case the income distribution is linear (see Pasinetti, 1977). Denoting these special vectors in this case by the symbols $l^{*}$ and $p^{*}$, respectively, we thus have

$$
p^{*}=(1+r) p^{*} A+w^{*} l^{*} .
$$

Now let us take a look at the right-hand side. $p^{*}$ being an eigenvector, the first term can be written as $(1+r) \mu_{1} p^{*} A_{1}$. Because $r=\left(1-w^{*}\right) R$, $l^{*}$ can be written as $\left(1-\mu_{1}\right) p^{*} A_{1}$. So, removing brackets, we obtain

$$
p^{*}=\mu_{1} p^{*} A_{1}+r \mu_{1} p^{*} A_{1}+u^{*}\left(1-\mu_{1}\right) p^{*} A_{1} .
$$


The columns of $A_{1}$, the first constituent matrix of $A$ (see footnote 6 ), are scalar multiples of the Perron-Frobenius eigenvector of $A$. Thus, $p^{*}$ can be decomposed in an intermediate inputs part $\mu_{1} p^{*} A_{3}$, an interest part $r \mu_{1} p^{*} A_{1}$, and a wage part $w^{*}\left(1-\mu_{1}\right) p^{*} A_{1}$, all expressed in terms of the columns of matrix $A_{1}$. Therefore, these columns can be viewed, also in this special case, as scalar multiples of a system standard of value of the type as discussed in Sect. 3 for the closed model. ${ }^{7}$

Note that our price model can be written as the price equation of a closed model with input coefficients matrix $\left(1 / \mu_{1}\right) A$. To see this, let us rewrite ( $\left.\mathrm{I}^{\prime \prime}\right)$. Using $\mu_{1} p^{*} A_{1}=p^{*} A$, we have

$$
p^{*}=p^{*} A+r p^{*} A+\frac{w^{*}\left(1-\mu_{1}\right)}{\mu_{1}} p^{*} A .
$$

After some manipulation, we then obtain the simplified form

$$
p^{*}=\frac{1}{\mu_{1}} p^{*} A
$$

So, we may conclude that this case is remarkably similar to the cases of Sect. 3.

The Standard system provides a case dual to the previous case of the special price system. We clearly have $A x^{*}=\mu_{1} x^{*}$. Thus $f^{*}=$ $\left(1-\mu_{1}\right) x^{*}$, and we obtain

$$
\begin{aligned}
x^{*} & =A x^{*}+\left(1-\mu_{1}\right) x^{*} \\
& =A x^{*}+\frac{1-\mu_{1}}{\mu_{1}} A x^{*} \\
& =\left(A+\frac{1-\mu_{1}}{\mu_{1}} A\right) x^{*} \\
& =\frac{1}{\mu_{1}} A x^{*} .
\end{aligned}
$$

So, total output in the Standard system also can be written in terms of

7 We observe that our standardizations imply that $A_{1}$, having been defined in terms of $p^{*}$ and $f^{*}$ (see footnote 6), equivalently can be defined in terms of $l^{*}$ and $x^{*}$. From $\left(1^{\prime}\right)$ we have $l^{*}=\left(1-\mu_{1}\right) p^{*} A_{1}=\left(1-\mu_{1}\right) p^{*}$. On the other hand, from the Standard system equation we have $x^{*}=A x^{*}+f^{*}=\mu_{1} x^{*}$ $+f^{*}$, which gives $f^{*}=\left(1-\mu_{1}\right) x^{*}$. So, $A_{1}=\left[f^{*} p^{*}\right]=\left[\left(1-\mu_{1}\right) x^{*}\right\} \times$ $\left.\left[\left[1 /\left(1-\mu_{1}\right)\right] l^{*}\right]\right]=\left[x^{*} l^{*}\right]$. 
a closed input-output model with input coefficients matrix $\left(1 / \mu_{1}\right) A$. Straightforwardly, we now have

$$
\begin{aligned}
x^{*} & =A_{1} x^{*} \\
& =\mu_{1} A_{1} x^{*}+\left(1-\mu_{1}\right) A_{1} x^{*} .
\end{aligned}
$$

So, again, the production of each good can be written in terms of the Perron-Frobenius eigenvector which then acts as a standard of value. The intermediate inputs then "use up" Standard commodities in the amounts indicated by the first term on the right-hand side of (14), for wages and profits then Standard commodities in the amounts indicated by the second right-hand term are available. Finally, we should notice that in $(1 ")$ for each sector of the economy, the proportions between the parts of its price going to intermediate outlays and to value added $\left(\mu_{1}\right.$ and $1-\mu_{1}$, respectively) are identical to the proportions of total output going to intermediate inputs and final demand in the whole economy. (The above observed duality does not mean, of course, that only $p=p^{*}$ is compatible with the Standard system equations, see further Sect. 5.)

We have not yet discussed income distributions proper. The literature tells us that if $l=l^{*}$, the relationship between $r$ and $w^{*}$ is linear. This also is the case if the real output system is the Standard system (as already observed by Sraffa). In other cases the wage profit tradeoff is complex, and generally nonlinear, the one exception apparently being the case, as claimed by Sraffa, where the Standard commodity is introduced as value standard, see, e.g., Pasinetti $(1977$, p. 88$)$. In the literature on Sraffa, there is no theoretical connection between these (three) cases. Below we shall derive one. Goodwin derives simple income distributions in terms of all eigenvectors of the input coefficients matrix. In terms of our proposed framework this immediately is seen to be very similar to Sraffa's approach.

\section{The Linear Wage Profit Trade-Off}

In this section we shall present two propositions to illustrate the role of eigenvectors as standards of value. We start with the real output model

$$
x=A x+f .
$$

Introducing prices we obtain

$$
p x=p A x+p f
$$


Income distribution theory tells us that

$$
p f=r p A x+w^{*} p f .
$$

So

$$
p x=p A_{x}+r p A x+w^{*} p f .
$$

We now focus on the links between the total output vector $x$ and the price vector $p$ in this model. Such connections have not received due attention in earlier work in this area. From (16), employing the spectral decomposition of the matrix of input coefficients, we have

$$
\begin{aligned}
p x= & p\left[\mu_{1} A_{1}+\mu_{2} A_{2}+\ldots+\mu_{n} A_{n}\right] x+ \\
& +r p\left[\mu_{1} A_{1}+\mu_{2} A_{2}+\ldots+\mu_{n} A_{n}\right] x+ \\
& +w^{*} p\left[I-\left(\mu_{1} A_{1}+\mu_{2} A_{2}+\ldots+\mu_{n} A_{n}\right)\right] x \\
= & p\left[\mu_{1} A_{1}+\mu_{2} A_{2}+\ldots+\mu_{n} A_{n}\right] x+ \\
& +r p\left[\mu_{1} A_{1}+\mu_{2} A_{2}+\ldots+\mu_{n} A_{n}\right] x+ \\
& \left.+w^{*} p\left[\left(1-\mu_{1}\right) A_{1}+\left(1-\mu_{2}\right) A_{2}+\ldots+\left(1-\mu_{n}\right) A_{n}\right)\right] x .
\end{aligned}
$$

(In the last step we have used $l=A_{1}+A_{2}+\ldots+A_{n}$ )

Excluding for the moment the case $w^{*}=1$, and, for reasons to become clear later on, the case $x=x^{*}$ (i.e., the Standard system case), we now have the following

Proposition 1: Consider the system (16). Then for $w^{*} \in[0,1)$

$$
\left\{\forall x: r=\left(1-w^{*}\right) R\right\} \Longleftrightarrow p=p A_{1} .
$$

\section{Proof:}

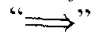

Let the eigenvectors of $A$ (see footnote 6 ) be normalized such that $w_{i} v_{i}=1$ and $w_{i} v_{j}=0$ for $\left.i \neq j\right) .{ }^{8}$ Then

$$
\text { and } \quad \begin{aligned}
& w_{i} A_{i}=u_{i} v_{i} w_{i}=w_{i} \\
& w_{i} A_{j}=w_{i} v_{j} w_{j}=0, \quad i \neq j .
\end{aligned}
$$

8 In Propositions 1 and 2, we employ the notation introduced in footnote 6. 
Now we have

$$
\begin{aligned}
p x= & p A x+r p A x+w^{*} p(I-A) x \\
\Longrightarrow \quad p x= & p\left(\sum_{i=1}^{n} \mu_{i} A_{i}\right) x+r p\left(\sum_{i=1}^{n} \mu_{i} A_{i}\right) x+ \\
& +w^{*} p\left(\sum_{i=1}^{n}\left(1-\mu_{i}\right) A_{i}\right) x \\
\Longrightarrow \quad p x= & p\left[\mu_{1}+r \mu_{1}+w^{*}\left(1-\mu_{1}\right)\right] A_{1} x+ \\
& +p\left(\sum_{i=2}^{n}\left[\mu_{i}+r \mu_{i}+w^{*}\left(1-\mu_{i}\right)\right] A_{i}\right) x .
\end{aligned}
$$

Using $r=\left(1-w^{*}\right) R$ and $R=\left(1-\mu_{1}\right) / \mu_{1}$, we obtain

$$
\mu_{1}+r \mu_{1}+w^{*}\left(1-\mu_{1}\right)=1 \text {. }
$$

which gives

$$
p x=p A_{1} x+p\left(\sum_{i=2}^{n} \Phi_{i}\left(w^{*}\right) A_{i}\right) x
$$

with

$$
\Phi_{i}\left(w^{*}\right) \equiv\left[\mu_{i}+r \mu_{i}+w^{*}\left(1-\mu_{i}\right)\right] .
$$

[Note that $\Phi_{i}\left(w^{*}\right)=1 \Longleftrightarrow w^{*}=1$.] So

$$
p\left(\sum_{i=2}^{n} A_{i}\right) x=p\left(\sum_{i=2}^{n} \Phi_{i}\left(w^{*}\right) A_{i}\right) x
$$

or

$$
p\left(\sum_{i=2}^{n}\left(\Phi_{i}\left(w^{*}\right)-1\right) A_{i}\right) x=0
$$

which must be true for all $x$. Writing $p$ as a function of $w^{*}$, we then have

$$
p\left(w^{*}\right)\left[\sum_{i=2}^{n}\left(\Phi_{i}\left(w^{*}\right)-1\right) A_{i}\right]=0, \quad w^{*} \in[0,1) .
$$


Generally, $p\left(w^{*}\right)$ can be written as a nontrivial combination of the $n$ bi-orthonormalized left-hand eigenvectors of $A$. Now write

$$
p\left(w^{*}\right)=\sum_{i=1}^{n} \beta_{i}\left(w^{*}\right) w_{i}
$$

and choose $w_{1}$ such that $\beta_{1}=1$. Then

$$
\begin{aligned}
\sum_{i=1}^{n} \beta_{i}\left(w^{*}\right) w_{i}\left[\sum_{i=2}^{n}\left(\Phi_{i}\left(w^{*}\right)-1\right) A_{i}\right] & =0, & & w^{*} \in[0,1) \\
\sum_{i=2}^{n} \beta_{i}\left(w^{*}\right)\left(\Phi_{i}\left(w^{*}\right)-1\right) w_{i} & =0, & & w^{*} \in[0,1) .
\end{aligned}
$$

Now each $\beta_{i}\left(w^{*}\right)=0(i=2, \ldots, n)$, for all $w^{*}$, because otherwise the zero vector can be written as a nontrivial linear combination of the $u_{i},(i=2, \ldots, n)$. This gives

$$
p\left(w^{*}\right)=w_{1} . \quad w^{*} \in[0,1),
$$

and with $w_{1}=w_{1} A_{1}$, we have $p=p A_{1}$. (Note that $w_{1}$ is the left-hand Frobenius eigenvector of $A$.)

$$
\text { "ఓ" }
$$

From (17), we have

$$
\begin{aligned}
p x= & p\left[\mu_{1}+r \mu_{1}+w^{*}\left(1-\mu_{1}\right)\right] A_{1} x+ \\
& +p\left(\sum_{i=2}^{n}\left[\mu_{i}+r \mu_{i}+w^{*}\left(1-\mu_{i}\right)\right] A_{i}\right) x .
\end{aligned}
$$

From $p=p A_{1}$ and $p A_{i}=0(i=2, \ldots, n)$ it follows that

$$
p x=\left[\mu_{1}+r \mu_{1}+w^{*}\left(1-\mu_{1}\right)\right] p x
$$

or

$$
1=\mu_{1}+r \mu_{1}+w^{*}\left(1-\mu_{1}\right)
$$

Substituting $\mu_{1}=1 /(1+R)$, we obtain (3) straightforwardly.

For the case $x=x^{*}$, we refer to the next proposition, which gives a dual result. We have 
Proposition 2: Consider again system (16). Then for $w^{*} \in[0,1)$ we have

$$
\left\{\forall p: r=\left(1-w^{*}\right) R\right\} \Longleftrightarrow x=A_{1} x
$$

Proof:

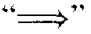

The proof is identical to the previous one up to

$$
p\left(\sum_{i=2}^{n}\left(\Phi_{i}\left(w^{*}\right)-1\right) A_{i}\right) x=0, \quad w^{*} \in[0,1)
$$

which now must be true for all $p\left(w^{*}\right) \neq p^{*}$. Now write $x$ as a nontrivial combination of the $n$ bi-orthonormalized right-hand eigenvectors of $A$ :

$$
x=\sum_{i=1}^{n} \alpha_{i} v_{i}
$$

So, with $p=\sum_{i=1}^{n} \beta_{i}\left(w^{*}\right) w_{i}$,

$$
\begin{aligned}
& \left(\sum_{i=1}^{n} \beta_{i}\left(w^{*}\right) w_{i}\right)\left(\sum_{i=2}^{n}\left(\Phi_{i}\left(w^{*}\right)-1\right) A_{i}\right) \times \\
& \times\left(\sum_{i=1}^{n} \alpha_{i} v_{i}\right)=0, \quad w^{*} \in[0,1) . \\
& \Longrightarrow \quad\left(\sum_{i=2}^{n} \beta_{i}\left(w^{*}\right) w_{i}\right)\left(\sum_{i=2}^{n}\left(\Phi_{i}\left(w^{*}\right)-1\right) A_{i}\right) \times \\
& \times\left(\sum_{i=2}^{n} \alpha_{i} v_{i}\right)=0, \quad w^{*} \in[0,1) . \\
& \Longrightarrow \quad\left(\sum_{i=2}^{n} \beta_{i}\left(w^{*}\right) w_{i}\right)\left(\sum_{i=2}^{n}\left(\Phi_{i}\left(w^{*}\right)-1\right) \alpha_{i} v_{i}\right)=0, \quad w^{*} \in[0,1) . \\
& \Longrightarrow \quad \sum_{i=2}^{n} \beta_{i}\left(w^{*}\right)\left(\Phi_{i}\left(w^{*}\right)-1\right) \alpha_{i}=0, \quad w^{*} \in[0,1) .
\end{aligned}
$$

With $\delta_{i}\left(w^{*}\right) \equiv \beta_{i}\left(w^{*}\right)\left(\Phi_{i}\left(w^{*}\right)-1\right)$, we now have 


$$
\sum_{i=2}^{n} \delta_{i}\left(w^{*}\right) \alpha_{i}=0, \quad w^{*} \in[0,1],
$$

so $\alpha_{i}=0, i=2, \ldots, n$. This implies $x=A_{1} x$.

"Е": Straightforward.

(For the case $p=p^{*}$, we refer to Proposition 1.) Proposition 1 thus tells us that if prices do not change if the wage rate or rate of profits change, the latter variables are linearly related according to (3). The case $w^{*}=1$ is the classic labor theory of value case. Proposition 2 essentially tells us that if any price vector should be allowed, only the case $x=x^{*}$ (i.e., the Standard system case) is possible. This, in our view, leads to the correct interpretation of the traditional linearity proof as reproduced in Sect. 2. As we see, this proof only tells us that if prices are given by (1), Eq. (3) is found if the output vector is that of the Standard system (because the price vector is post-multiplied by $x^{*}$ ). Consequently, the traditional proof concerns only the "—" part of Proposition 2.

\section{The General Case}

Proposition 1 tells us that to obtain a linear relation between $r$ and $w^{*}$ in input-output systems other than the Standard system, prices must satisfy

$$
p=p A_{1} .
$$

That is, abiding by our symbols and standardizations, we must have $p=p^{*}$. We have seen that this implies that the vector of direct labor input coefficients is a left-hand Perron-Frobenius eigenvector of $A$. In that case we have

$$
p^{*}=\mu_{1} p^{*} A_{1}+r \mu_{1} p^{*} A_{1}+w^{*}\left(1-\mu_{1}\right) p^{*} A_{1} .
$$

The price of each good consists of three components, each nicely expressed in terms of the Standard commodity in the sense defined earlier. Proposition 2 is the case of the Standard system. We have $x=x^{*}$ and for all (properly standardized) price vectors we essentially have the same relation.

By now, we have rewritten the two special cases which were introduced in Sect. 4 in terms of our new tool. However, the scope of Sraffa's work, of course, is much broader, discussing general results. 
Sraffa affirms that "imposing" the Standard commodity generates a linear income distribution mechanism for all input-output systems. In this respect, we should realize that up to now we have not "imposed" anything. We only have shown that in two special cases where (3) is valid, the price equation can be interpreted in terms of the Standard commodity. This does not tell us anything yet about arbitrarily chosen systems. So, let us turn now to the general case.

Equation (18) tells us that all we need is a price vector equal to a transformation of itself, the transformation matrix being $A_{1}$. Now let us consider again the general price equation (1) and consider the transformation:

$$
p^{*}=p A_{1}=\left[(1+r) p A+w^{*} l\right] A_{1} .
$$

We see that this transformation results in the one price vector, i.e., $p^{*}$, which - as we have seen - perfectly does the job. Straightforwardly, we find

$$
\begin{aligned}
p^{*} & =p A A_{1}+r p A A_{1}+w^{*} l A_{1} \\
& =\mu_{1} p^{*} A_{1}+r \mu_{1} p^{*} A_{1}+w^{*}\left(1-\mu_{1}\right) p^{*} A_{1},
\end{aligned}
$$

which equals (1"). Now, how to interpret this? Let us take a look first at the intermediate inputs part. Instead of the term $p A$ we here encounter the term $\mu_{1} p^{*} A_{1}$. That is, the transformation imputes to each industry inputs precisely in the proportions of the Standard commodity. The income part that is imputed to each industry equally is given in terms of (the value of) the Standard commodity. The value proportion between intermediate inputs part $\mu_{1} p^{*} A_{1}$ and income part $\left(1-\mu_{1}\right) p^{*} A_{1}$ is $\mu_{1} /\left(1-\mu_{1}\right)$. As we might have expected, we here find the same proportions as between the intermediate inputs part and the income part for the Standard system: $p A x^{*} / p f^{*}=\mu_{1} p x^{*} /\left[\left(1-\mu_{1}\right) p x^{*}\right]=$ $\mu_{1} /\left(1-\mu_{1}\right)$. So, on the industry level the proportions of the macro system (i.e., the Standard system) are repeated over and over again, as in the earlier cases of Sects. 3 and 4. (19) shows that by imposing a transformation of prices, we "force" the system to a rearrangement to the effect that the fractions of each price going to intermediate outlays and the value-added part are identical to the fractions in the special price system discussed in Sect. 4. That, as we have seen, is sufficient to guarantee a linear income distribution relation.

Above we have introduced transformed prices. An obvious question is: Is there a need to transform the real system? Actually, we still have to see to what kind of transformed commodities the new prices correspond, and how the - appropriately adapted - new input matrix 
looks like. Unfortunately, very little work on the type of approach we are dealing with is available. We should note that the situation is quite different from a simple change of units (ounces into pounds etc.) such as described in Fisher's (1965) study. For example, instead of working with a full rank matrix, as in Fisher's case, we now have to work with a rank one matrix (i.e., $A_{1}$ ) as our transformation matrix. Fortunately, on the other hand, the structure of the system is such that this second step, the creation of the new commodities, is not necessary. To see this, let us postmultiply (19) by $x$, the output vector:

$$
p A_{1} x=\left[(1+r) p A+w^{*} l\right] A_{1} x .
$$

Because $A_{1}$ is idempotent, we may also write

$$
p A_{1} A_{1} x=\left[(1+r) p A+w^{*} l\right] A_{1} A_{1} x
$$

Equation (21) can be interpreted as the product of transformed prices $\left(p A_{1}\right)$ and goods $\left(A_{1} x\right)$ transformed via premultiplication of the output vector by $A_{1}$. Premultiplication by $A_{1}$ means that the new, first good is a weighted average of the old goods, the weights being given by the first row of $A_{1}$, similar interpretations being available for the remaining other goods. By this transformation, clearly, all input proportions become equal, thus eliminating all sectoral differences. However, as we easily observe by comparing (20) and (21) - which give exactly the same result - the particular weights as given by $A_{1}$ have the extremely useful property that we can work with the transformed prices, without having to transform the real output system correspondingly. Because of $A_{1}$ being idempotent we can successively introduce a transformation that only involves a transformation of the price system.

Earlier Professor Goodwin already drew our attention to the fact that transformations may be very useful in studying distribution problems. In the next section we shall discuss in particular the common framework with Goodwin's results.

\section{Goodwin's Transformations}

Above we have seen that in the general case, we only obtain a linear income distribution if we employ transformed prices. The transformation meant that instead of working with prices $p$ given by (1), we now work with prices satisfying the transformed Eq. (19).

It will be interesting to explore the relation with a distribution model proposed by Goodwin $(1974,1976,1983)$. Goodwin's approach 
is quite unlike other modelling efforts because a transformation of axes is involved. As such, Goodwin's work has a rather unique place in distribution theory. A number of publications have recently appeared where Goodwin's normalized general coordinates are compared with Pasinetti's vertically integrated sectors method, see Pasinetti (1990) or Cozzi (1990). Next to a number of similarities also a number of significant differences were noted. Part of these were a result of the fact that Goodwin removes all sectoral interdependence while Pasinetti explicitly retains as much structure as possible. In terms of our framework, a major difference with these comparisons is that neither Pasinetti nor Cozzi were trying to formulate a common mathematical framework. Now let us return to Goodwin's work and consider the following typical equation (1976, p. 582):

$$
[I-(1+g) A] Q_{0}=\{(1+g) \alpha\} C_{0} .
$$

Here $Q_{0}$ is the output vector, $C_{0}$ the final demand or consumption vector, $g$ the growth rate, $\alpha$ a scale factor, and $A$ the matrix of input coefficients, supposedly diagonable. (For our purposes it does not matter that this equation is formulated in terms of investment and consumption, rather than profits and wages. By giving $\alpha$ and $g$ the values 1 and 0 , respectively, and by writing $x$ for $Q_{0}$ and $f$ for $C_{0}$, we obtain the standard formula (2). For Goodwin's price system, naturally, a similar transformation exists. Because in this paper we are only interested in the methodology that leads to the desired simplification, the fact that the modet is rather of the Marxian than the Sraffian type need not concern us here.)

Goodwin proposes a transformation based on the orthonormal matrix $H$ of eigenvectors given by

$$
H A H^{-1}=\hat{\mu}
$$

where $\hat{\mu}$ is the diagonal matrix of the eigenvalues of $A$. From (16) we notice that the columns of $H^{-1}$ are the right-hand eigenvectors and the rows of $H$ the left-hand eigenvectors of $A$; the property $H^{-1} H=I$ guarantees normalization. Introducing

$$
H^{-1} Q \equiv Q_{0}
$$

and

$$
H^{-1} C \equiv C_{0}
$$


Goodwin obtains via

$$
\begin{aligned}
& H[I-(1+g) A] Q_{0}=\{(1+g) \alpha\} H C_{0} \\
& \Longrightarrow \quad H[I-(1+g) A] H^{-1} H Q_{0}=\{(1+g) \alpha\} H C_{0} \\
& \Longrightarrow \quad[I-(1+g) \hat{\mu}] H Q_{0}=\{(1+g) \alpha\} H C_{0}
\end{aligned}
$$

the simplification

$$
[I-(1+g) \hat{\mu}] Q=\{(1+g) \alpha\} C .
$$

As observed by Goodwin, this transformation defines $n$ distinct composite commodities. A remarkable fact of this system is that each commodity is produced entirely out of inputs of its own kind. Wages and investment similarly consist only of this same commodity. Thus, simple linear income trade-offs are a property of this system. Goodwin comments that his device will ordinarily involve negative and complex quantities which will present serious interpretatory problems. (Regarding this, Goodwin observes that we always can go back to our original coordinates for an economic interpretation. This, of course, is a possibility, but we should remark that then the whole point of the transformation would be lost.) Also, as already observed by Goodwin, by operating with all eigenvectors in this particular manner (i.e., with the nonsingular eigenvector matrix $H$ ), the desired simplification of the income distribution process is obtained by sacrificing the entire intermediate structure.

Now let us see what the connection is with our work. From (23) we have

$$
A=H^{-1} \hat{\mu} H .
$$

Therefore

$$
A=\mu_{1}\left(h_{.1}^{-1} h_{1}\right)+\mu_{2}\left(h_{.2}^{-1} h_{2}\right)+\ldots+\mu_{n}\left(h_{. n}^{-1} h_{n}\right)
$$

with $h_{. i}^{-1}$ the $i$-th column of $H^{-1}$ and $h_{i}$ the $i$-th row of $H$. Thus (26) is nothing but the familiar spectral decomposition of $A$, with

$$
h_{. i}^{-1} h_{i}=A_{i} .
$$

Instead of employing matrix $H$ which leads to (24), in our approach we premultiply Eq. (22) (or any other equivalent real output equation) by $A_{1}$ and use $A_{1} A=\mu_{1} A_{1}$ and $\left(A_{1}\right)^{2}=A_{1}$. Starting from Goodwin's 
Eq. (22), our approach can be written in terms of the following sequence of steps:

$$
\begin{array}{rlrl}
A_{1}[I-(1+g) A] Q_{0} & =\{(1+g) \alpha\} A_{1} C_{0} \\
& \Rightarrow & {\left[A_{1}-(1+g) \mu_{1} A_{1}\right] A_{1} Q_{0}} & =\{(1+g) \alpha\} A_{1} C_{0} \\
& \Rightarrow & {\left[I-(1+g) \mu_{1} I\right] A_{1} Q_{0}} & =\{(1+g) \alpha\} A_{1} C_{0} .
\end{array}
$$

Writing $x=Q_{0}$ and $f=C_{0}$, we obtain

$$
\left[I-(1+g) \hat{\mu}_{1}\right] A_{1} x=\{(1+g) \alpha\} A_{1} f
$$

with $\hat{\mu}_{1}=\mu_{1} I$. (Substituting $g=0$ and $\alpha=1$, and premultiplying by $p$, we obtain our basic formulas for $r=0$.)

Thus, by concentrating on the constituent matrices $A_{i}$ (instead of on $H$ ), in our approach we were able to retain more structure in the intermediate part than Goodwin in his approach. In fact, the structure retained is the rank one matrix $A_{1}$, the columns of which are scalar multiples of the Standard commodity. The same linear trade-off now is valid for each good, and awkward negative and complex entries are not possible. Of course, if, instead, we would concentrate on the matrices $A_{i}$ ( $\left.i=2, \ldots, n\right)$, we would have obtained linear income distributions involving negative or complex quantities of the type also referred to by Goodwin.

\section{Further Remarks and Conclusion}

We should remark that there is some room for generalization. For example, in this paper we have chosen $A_{I}$ as our transformation matrix. Other possibilities do exist, though. If we select as transformation matrix

$$
T=\left[f p^{*}\right]
$$

with $f$ an arbitrary net output vector and again impose the standardizations $p^{*} f=1$, consumer preferences are reflected in the colums of the transformation matrix $T$, i.e., in the then adopted value standard. Such transformations are always possible as the crucial left-hand eigenvector of $T$ (being also the left-hand eigenvector of $A$ ) is determined by the economy's technology. The possibility of extending the framework in this direction may provide an additional difference between our approach and Goodwin's. In our approach, it is easily shown that any bundle $k$ satisfying $p T$, with $T=\left[k p^{*}\right]$ and $p k=1$, can be a system numeraire in the sense we have defined it. In Goodwin's system this 
property of linear income trade-offs is difficult to locate. This property of our system may also provide an interpretation of Sraffa's remark (1960, sect. 43) regarding the role of the specific proportions of the Standard system. It also answers much of Burmeister's criticism of the Sraffian framework, presented in a number of publications. For example, in Burmeister (1984) it is pointed out that large weights should be given to commodities that are never consumed by human beings. As shown by the above, this need not be the case at all. For additional criticism on Burmeister's interpretation of Sraffa, in line with our approach, see especially Kurz and Salvadori $(1987,1993)$.

A further remark seems appropriate here. In the context of our framework, it is interesting to note that Quandt (1961), in an early review of Sraffa's work, already pointed out certain similarities with Leontief's closed model. In our above described approach, Sraffa's system does indeed have a number of properties in common with Leontief's closed model. The special cases discussed in Sect. 4 are seen to already provide the basic equations of the development in our later sections. In this respect, Quandt's insights regarding this similarity (on which, unfortunately, he did not elaborate much) may be called quite remarkable. (For comments on Quandt's observation, see also Levine, 1974.) Other models are easily seen to fit the same framework. For example, also the equilibrium case of Leontief's closed dynamic model (Bródy, 1970, chap. 1.2) can be analyzed in this way (Steenge, 1990a).

Above we have presented a new look at certain well-established results in distribution theory. We have shown that earlier approaches put forward by Sraffa and Goodwin can be analyzed in terms of a unifying framework. In developing this framework we have established that eigenvectors really can be viewed as "instruments," i.e., they can be imposed on the system in such a way that they really become standards of value, i.e., commodity bundles the amounts of which imputed to each individual good in the system determine the price of that particular good. The way to proceed appeared to be via a rank one matrix which then plays the central role in methodologies involving eigenvectors as the Leontief inverse or its derivatives in traditional analysis. Most likely the above framework can be further simplified and applied to other areas such as models allowing for joint production. Nevertheless, we hope to have shown the potential of modelling the role of eigenvectors in terms of certain transformations in this field of research. 


\section{Acknowledgements}

Earlier versions of this paper have been presented at the European Economic Association meeting in Cambridge, UK, 31 August - 2 September 1991, and at the Fourth Annual Workshop on The Wealth of Nations in Economic Theory. Gargnano, 18-20 September 1993. The author is indebted to a number of people for comments and suggestions. In particular he would like to thank Gérard Duménil, Harald Hagemann, Paul Konijn, Heinz Kurz, Dominique Lévy, Gerhard Meinen, Luigi Pasinetti, and Michael Sonis. Two anonymous referees are acknowledged for valuable comments and encouragement.

\section{References}

Abraham-Frois, A., and Berrebi, E. (1979): Theory of Value, Prices and Accumulation. Cambridge: Cambridge University Press.

Bródy, A. (1970): Proportions, Prices and Planning. Amsterdam: North-Holland.

Burmeister, E. (1984): "Sraffa, Labor Theories of Value, and the Economics of Real Wage Determination." Journal of Political Economy 92: 508-526.

Cozzi, T. (1990): "A Comparison Between Goodwin's Normalised General Coordinates and Pasinetti's Vertical Integration Method." In Nonlinear and Multisectoral Macrodynamics, Essays in Honour of Richard Goodwin. edited by K. Velupillai. London: Macmillan.

Fisher, F. M. (1965): "Choice of Units, Column Sums and Stability in Linear Dynamic Systems with Nonnegative Matrices." Econometrica 33: 445450 .

Goodwin, R. M. (1974): "The Use of Normalized General Coordinates in Economic Planning." In Economic Theory and Planning, Essays in Honour of A. K. Das Gupta, edited by A. Mitra. Calcutta: Oxford University Press. (1976): "Use of Normalized General Coordinates in Linear Value and Distribution Theory." In Advances in Input-Output Analysis, Proceedings of the Sixth International Conference on Input-Output Techniques, Vienna. edited by K. R. Polenske and J. V. Skolka. Cambridge, MA: Ballinger.

- (1983): Essays in Linear Economic Structures. London: Macmillan.

Konijn, P. J. A., and Steenge, A. E. (1995): "Compilation of Input-Output Data from the National Accounts." Economic Systems Research 7: 31-45.

Kurz, H. D., and Salvadori, N. (1987): "Burmeister on Sraffa and the Labor Theory of value: a Comment." Journal of Political Economy 95: 870-881. (1993): "The 'Standard Commodity' and Ricardo's Search for an 'Invariable Measure of Value"." In The Dynamics of the Wealth of Nations: Growth, Distribution and Structural Change, Essays in Honour of Luigi Pasinetti, edited by M. Baranzini and G. C. Harcourt. London: Macmillan. Levine, A. L. (1974): "This Age of Leontief ... and Who? An Interpretation." Journal of Economic Literature 12: 872-881. 
Pasinetti, L. L. (1977): Lectures on the Theory of Production. New York: Columbia University Press.

(1990): "Normalised General Coordinates and Vertically Integrated Sectors in a Simple Case." In Nonlinear and Multisectoral Macrodynamics. Essays in Honour of Richard Goodwin, edited by K. Velupillai. London: Macmillan.

Quandt, R. E. (1961): "Review of 'Production of Commodities by Means of Commodities". Journal of Political Economy 69: 500.

Seton, F. (1985): Cost, Use and Valae: the Evaluation of Performance, Structure, and Prices Across Time, Space, and Economic Systems. Oxford: Clarendon Press.

Sraffa, P. (1960): Production of Commodities by Means of Commodities. Cambridge: Cambridge University Press.

Steenge, A. E. (1986): "Saaty's Consistency Analysis: an Application to Problems in Static and Dynamic Input-Output Analysis." Socio-Economic Planning Sciences 20: 173-180.

(1990a): "On the Complete Instability of Empirically Determined Dynamic Leontief Models." Economic Systems Research 2: 3-16.

(1990b): "The Commodity Technology Revisited: Theoretical Basis and an Application to Error Location in the Make-Use Framework." Economic Modelling 4: 376-387.

Address of author: Albert E. Steenge, Faculty of Public Administration and Public Policy, University of Twente, P. O. Box 217, NL-7500 AE Enschede. The Netherlands. 
Copyright of Journal of Economics is the property of Springer Science \& Business Media B.V.. The copyright in an individual article may be maintained by the author in certain cases. Content may not be copied or emailed to multiple sites or posted to a listserv without the copyright holder's express written permission. However, users may print, download, or email articles for individual use. 